\title{
Cucumber appearance quality detection under complex background based on image processing
}

\author{
Haijian Ye*, Chengqi Liu, Peiyun Niu \\ (College of Information and Electronics Engineering, China Agricultural University, Beijing 100083, China)
}

\begin{abstract}
Cucumber fruit appearance quality is an important basis of growth status. In order to improve the quality detection accuracy and processing efficiency of cucumber color image under complicated background, an improved GrabCut algorithm was proposed to extract the cucumber boundary. Firstly, including pixel size normalization, rectangular box set and scale image resolution, pretreatments of cucumber image were adopted to reduce the iteration times and operation time of GrabCut algorithm. Then, the Gaussian mixture model was chosen to find out the possible prospect of target region and background region in the preprocessed rectangular frame on the preliminary modeling. Meanwhile, by the optimization of $K$-means cluster to the initial GMM model, the effective target area was extracted. Finally, the whole image noise and serrated boundary was removed by morphological operations to segment the outline of the complete target prospects with appropriate structure size. And then the cucumber appearance quality detection instrument was designed to extract the texture and shape features exactly, so that it could obtain cucumber appearance quality and evaluate its growth effectively. With the segmentation experiments by almost 300 cucumber original images from greenhouse in Shandong Province, the results showed that the improved GrabCut algorithm could effectively extract the complete and smooth boundary of cucumber. With relatively high segmentation evaluation index, the precision was $93.88 \%$, the recall rate was $99.35 \%$, the $F$-Measure reached $96.53 \%$, and the misclassification error was controlled at minimum 5.84\%. The average running time was shortened to $1.4023 \mathrm{~s}$. The comparison results showed that the improved GrabCut algorithm was the best, followed by the SLIC and traditional GrabCut method. Cucumber appearance quality detection instrument could also extract more accurate feature parameters. And it could meet the basic growth condition assessment by automatic image processing.
\end{abstract}

Keywords: cucumber, complicated background, quality detection, image processing, GrabCut

DOI: $10.25165 /$ j.ijabe.20181104.3090

Citation: Ye H J, Liu C Q, Niu P Y. Cucumber appearance quality detection under complex background based on image processing. Int J Agric \& Biol Eng, 2018; 11(4): 193-199.

\section{Introduction}

With the application of agricultural intelligence and refined technology, more accurate quality testing of the crops is required. Fresh fruits and vegetables contain rich essential substances which play an active role in improving the health status of people ${ }^{[1,2]}$. Regarding to detecting the rich information of Cucumber image, most of the existing researches are based on the laboratory and other simple environment ${ }^{[3]}$. Under the complex background of the fruit stem and leaf-light-stent, shade, uneven illumination and overlapping become the main factors of seriously affect the recognition accuracy and efficiency. As the final product of the plant and the direct demand of people, the growth condition of cucumber and the appearance quality of the fruit directly affected the yield formation and the farmers' income. Therefore, it is of great significance to evaluate the appearance quality and evaluate the growth of cucumber.

Image recognition is one of the necessary steps to achieve accurate detection of cucumber quality. The recognition algorithm has long been a research hotspot and difficulty in the

Received date: 2017-09-15 Accepted date: 2018-04-02

Biographies: Chengqi Liu, Master candidate, research interests: image processing, Email: 1835839033@qq.com; Peiyun Niu, Master candidate, research interests: image processing, Email: 815465347@qq.com.

*Corresponding Author: Haijian Ye, Professor, research interest: image processing and big data. College of Information and electrical engineering, China Agricultural University, Beijing 100083, China. Tel: +86-13910674154, Email: hjye@cau.edu.cn. field of image processing and computer vision. At present, the Graph cut algorithm proposed by Boykov ${ }^{[4]}$ is one of the most effective interactive segmentation methods. The GrabCut algorithm proposed by Rother ${ }^{[5]}$ has simplified the interaction of traditional image segmentation, and the effect is better than the others. Subsequently, the literatures [6-11] had improved the GrabCut algorithm in different ways. However, the existing interactive graph cut algorithms are still not ideal for the high similarity of the cucumber image. The complicated iterative solving process of the GMM model, the great waste in algorithm segmentation estimation and its dependence on unstable human interaction, are key points to many scholars' research for improvement $^{[12-14]}$.

Quality detection are mostly based on chemical and spectral techniques, this approach brings high testing precision, but also accompanied by high cost, difficult operation, disadvantages of long testing period or destruction of test sample quality. Several methods have been commonly applied in the field of the evaluation of preservation quality, such as wavelet domain, analytic hierarchy process and principal component analysis, quantum genetic fuzzy neural network, and particle clustering ${ }^{[15-23]}$. They were used to detect agricultural and livestock products. However, it has some limitations; commonly algorithms are not very good in solving cucumber quality detection under complicated background.

This paper studies greenhouse autumn cucumber under complicated background as the research object. Through the comparison and analysis of eight segmentation algorithms, as the optimal identification of cucumber fruits, an improved algorithm of 
GrabCut is selected to integrate cucumber appearance quality detection instrument. On the premise of Improving the recognition accuracy and efficiency, it extracts more accurate shape feature and texture feature parameters. According to the quality criteria, this paper realizes autumn cucumber quality detection ultimately.

Cucumber quality detection is presented in this paper. The research results are:

1) On the cucumber image recognition, the traditional GrabCut algorithm is improved. While keeping the core of GMM, the pre-segmentation fits the target area by external rectangular box to replace unstable human interaction. The data processing of algorithm is reduced by the size scaling. Mathematical morphology is used to replace Border Matting technology of GrabCut.

2) On the cucumber quality detection, cucumber appearance quality detection instrument was designed, integrated with the improved GrabCut algorithm, and combined with morphology and gray level co-occurrence matrix ${ }^{[24]}$, to solve the shape features and texture features extraction of cucumber.

3) Combined with cucumber grade standard, the relative weights of the key characteristic parameters are set, and the accurate grading of cucumber is completed.

\section{Materials and methods}

According to Figure 1, the flowchart of cucumber quality detection is used to improve the research and experiment comparison.

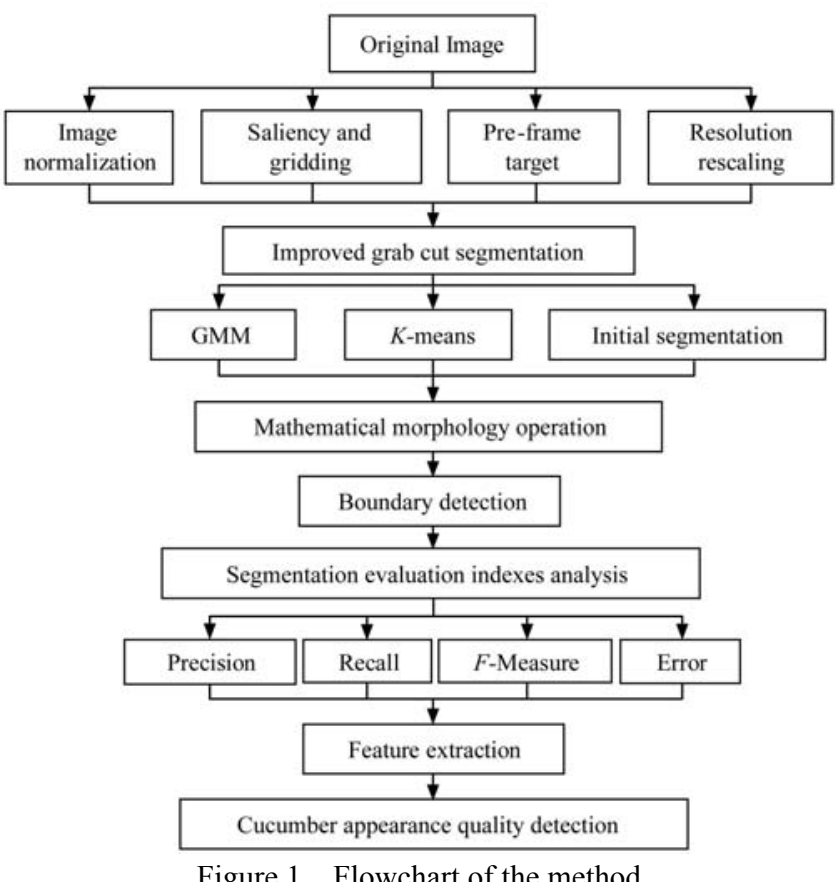

\subsection{Image capture and environment}

In this study, the cucumber image acquisition date were conducted from November 2016 to March 2017, from Qingdao greenhouses in Shandong province. Autumn cucumbers are almost wholesale and mature before picking. Image acquisition device are SLR cameras which is Canon 700d and smartphone of the rear 13 million pixels (HuaWei Honor Chang Play 4x, 267 dpi). This paper chooses MATLAB R2014a and OpenCV2.4.13 (platform for Visual Studio2015) as programming tools to realize image segmentation experiments. The configuration of the computer used in the experiment: Intel (R) Core (TM) i7-4720HQ
CPU@2.60 GHz, 16 GB ddr, Windows 7 (64 bit).

\subsection{Research status}

Traditional GrabCut algorithm usually requires the user to calibrate a rectangular box called the Region of Interest $(\mathrm{ROI})^{[25]}$. The outside and inside areas are initialized respectively to the background and the possible target area. This method turns the traditional graph cut interactions into non-interactions. The manual calibration $s-t$ terminal nodes ${ }^{[26]}$ shown in Figure 2 a can be omitted to improve the recognition accuracy and efficiency.

However, whether the recognition result is good or bad still depends on the artificial setting of ROI. It has a great deal of dependence. Once it appears with incomplete frame selection, as shown in Figure 2b, it would directly set target part as background. And as shown in Figure 2c, it appears over-complete frame selection. The algorithm will increase run time and over split occurred.

In addition, there is a variety of complex background in the actual planting. Some typical complicated background as shown in Figure 2d: (1) the illumination problem, (2) the overlapping problems, (3) the stem and leave problem and (4) the color problems, etc. The existing segmentation methods are difficult to one-time solve these problems and need a second division with artificial supplementary information, even it will lead to a drop in the recognition accuracy and efficiency. As a result, more improvements to the traditional GrabCut algorithm are necessary.

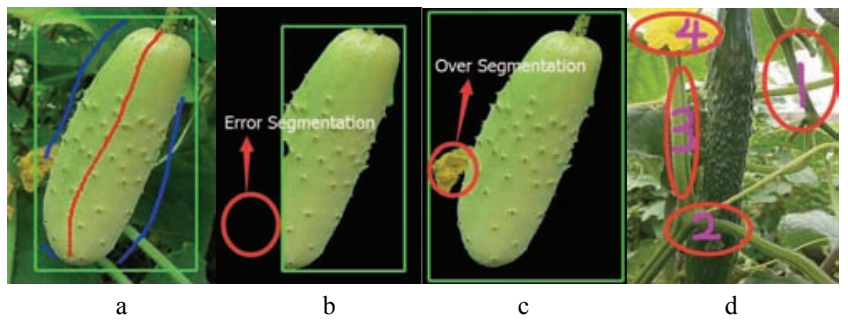

Figure 2 GrabCut by interaction manually

\subsection{Improved GrabCut algorithm}

\subsubsection{The pre-segmentation of cucumber}

On the premise of nondestructive identification, image can be further normalized completely. The smaller pixel level will be easier for image contour extraction. Firstly, $960 \times 1280$ pixels images are normalized to $320 \times 420$ pixels images. As shown in Figure $3 \mathrm{a}, 14 \times 14$ pieces of meshing is operated, and each small grid is preassigned as the foreground and background. Combining with significant analysis ${ }^{[27,28]}$ in Figure 3b, foreground target can be pre-selected. Due to the complex background, image target is not easy to be automatically selected. The selected rectangle box can appear deviated or incomplete box as shown in Figure 3c. It will require a series of displacement transformation. After fine-tuning made by amplification, narrowing and moving, rectangular box can fit goals external rectangle theoretically as shown in Figure 3d. Finally, the test image resolution is zoomed again, and the outside background pixel of the box can be adjusted to zero as shown in Figure 3e. It can greatly reduce the amount of data processing and prepare for next step of the GrabCut segmentation.

\subsubsection{Targeted cucumber extraction}

It removes the operation that GrabCut algorithm allows incomplete annotations after the last section, and it becomes a non-interactive method. In addition, it becomes possible to extract the targeted cucumber with a single GrabCut segmentation without affecting the segmentation effect. Then, the hybrid Gaussian model which has RGB 3-channel is used to select the 
target and build the background model, so that the segmentation estimation and model parameter learning can be carried out in one segmentation to achieve optimal goal.

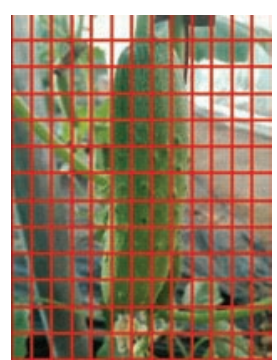

a

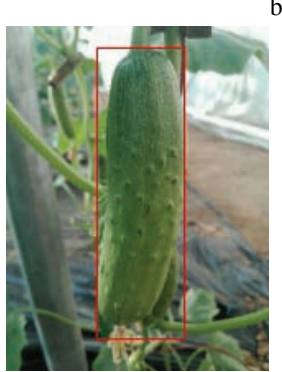

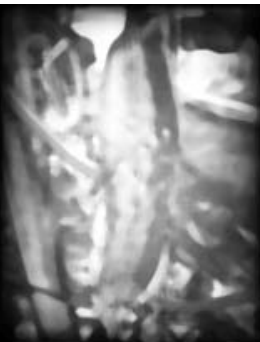

b

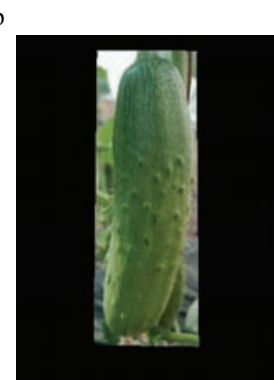

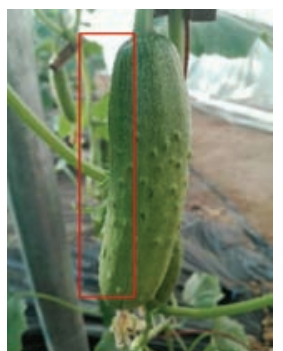

$\mathrm{c}$

Figure 3 ROI estimation

The GMM energy function of the structure is:

$$
E(\underline{\alpha}, k, \underline{\theta}, z)=U(\underline{\alpha}, k, \underline{\theta}, z)+V(\underline{\alpha}, z)
$$

where, $\underline{\alpha}$ is Differentiate vector for foreground and background (value 0 means for background, and value 1 means for foreground); $k$ is the model component of GMM; $\underline{\theta}$ is the unknown parameter; $z$ is image grayscale array.

In Equation (1), $U$ is defined as the color data item:

$$
\begin{gathered}
U(\underline{\alpha}, k, \underline{\theta}, z)=\sum_{n} D\left(\alpha_{n}, k_{n}, \underline{\theta}, z_{n}\right) \\
D\left(\alpha_{n}, k_{n}, \underline{\theta}, z_{n}\right)=-\log p\left(z_{n} \mid \alpha_{n}, k_{n}, \theta\right)-\log \pi\left(\alpha_{n}, k_{n}\right)
\end{gathered}
$$

where, $p(\cdot)$ is Gaussian probability distribution, $\pi(\cdot)$ is mixed weight coefficient.

In Equation (1), $V$ is defined as the smooth term (penalty item):

$$
V(\underline{\alpha}, z)=\gamma \sum_{(m, n) \in C}\left[\alpha_{n} \neq \alpha_{m}\right] \exp \left\{-\beta\left\|z_{m}-z_{n}\right\|^{2}\right\}
$$

where, $\gamma$ is the penalty factor, $\beta=\left(2\left\langle\left(z_{m}-z_{n}\right)^{2}\right\rangle\right)^{-1}$ is a constant.

The main steps of improved GrabCut algorithm are as follows:

1) Initializing outside background pixel $\mathrm{TB}$ of the rectangular box (the value of $\underline{\alpha}$ has already been set 0 during the pre-segmentation), and regarding inside of the rectangular box as a possible target pixel TU (the value of $\underline{\alpha}$ has already been set 1 during the pre-segmentation).

2) Distributing Gaussian component to each pixel $n$, and establishing $k$ (generally $k$ takes 5) GMM component models to the possible foreground and background of image.

3) For a given image data $z$, optimizing the unknown parameter $\underline{\theta}$ of GMM, namely the mean, covariance, and weight.

4) By analyzing the energy item, mapping and getting the weights of $t$-links and $n$-links, then Max flow/Min cut algorithm is used to estimate segmentation.

5) Repeat steps (2) to (4), until it gets convergence.

After zoom-processing of image resolution in the acceptable range ( 0.3 to 0.8 times), the efficiency can be enhanced greatly, as shown in Figures $4 \mathrm{a}$ and $4 \mathrm{~b}$ whose zoom is 0.8 times. Coupled with the pre-segmentation stage, algorithm can significantly lower internal number of iterations. The final nondestructive restores to the initial segmentation, Figure 4c. However, as shown in Figure 4d, the algorithm's own Border Matting technology which exists serrated edge details remains to be solved in the next step of morphology ${ }^{[29]}$ smoothing.

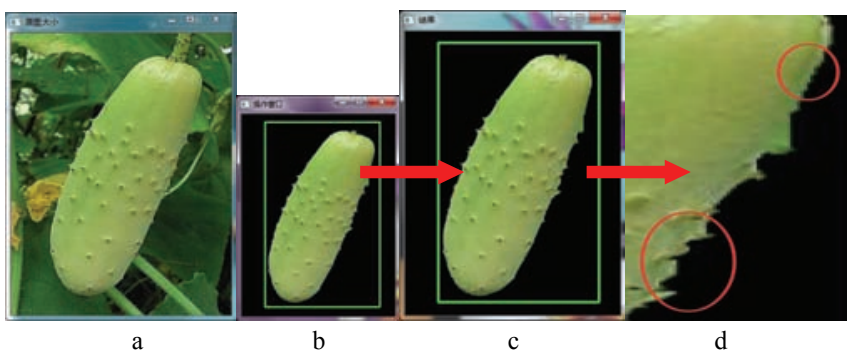

Figure 4 Segmentation of improved GrabCut in one cut

\subsubsection{Morphology processing}

The GrabCut algorithm uses Border Matting technology to calculate the continuous value in the strip surrounding the hard division boundary and obtains the ideal segmentation effect. However, complex background will affect the segmentation effect of Border Matting. As a result, mathematical morphology algorithm is used to replace Border Matting technology for further smoothing boundary and small noise. Choosing appropriate extraction radius, shape and structure, to improve efficiency at the same time get close to the result of the sample, as shown in Figure 5. The artificial segmentation binary diagram is shown in Figure 5a. The areas which is less than the target of $P$ (the structure element is 5) are removed by mathematical morphology method. After corrosion operation, it will remove the rough boundary slight noise but internal holes as shown in Figure 5b. After using the filling function and inflation as shown in Figure $5 \mathrm{c}$, and then to canny operator edge detection as shown in Figure $5 \mathrm{~d}$, it could get the ideal result by choosing appropriate extraction radius.

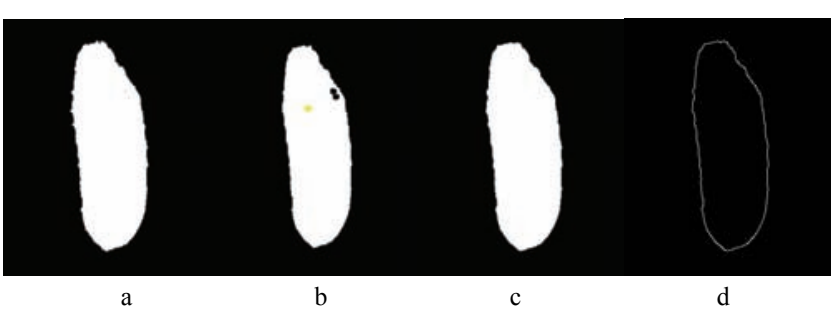

Figure 5 Morphology processing

\subsubsection{Improvement measures}

Improvement measures to the old GrabCut method formulated in this paper are mainly as follows. Firstly, external rectangular box of the target area is used to replace unstable human interaction of pre-segmentation. Secondly, properly adjusted size of image resolution is used to reduce the amount of data. Thirdly, morphological method is used to replace the Border Matting techniques of graph cut algorithm to solve the problem of target boundary roughness, so as to realize the improvement of GrabCut algorithm. As we mentioned in Section 2.3.1, we try to make the ROI calibration more accurate, to abandon the human interaction. Some rectangular boxes are not accurate in the pre-calibration process, so we choose the second best which make the rectangular box not too close to the target, just calibrate it in a proper extent. Then it can basically realize automation without human inputs.

\section{Experiment and results}

In order to further verify the reliability and stability of the identification method, this paper carries on experimental contrast of eight algorithms to verify the results as shown in Figure 6. At the 
same time, the image recognition evaluation indexes are shown in Figure 7. Including the Precision, the Recall rate, $F$-Measure and Error rate, it has carried on the quantitative analysis of comprehensive evaluation index which are shown in Table 1. And Table 2 shows the statistics of running time (s), as well as Figure 8 shows average running time visualization.

$$
\begin{aligned}
& P=\frac{T P}{T P+F P} \\
& R=\frac{T P}{T P+F N}
\end{aligned}
$$
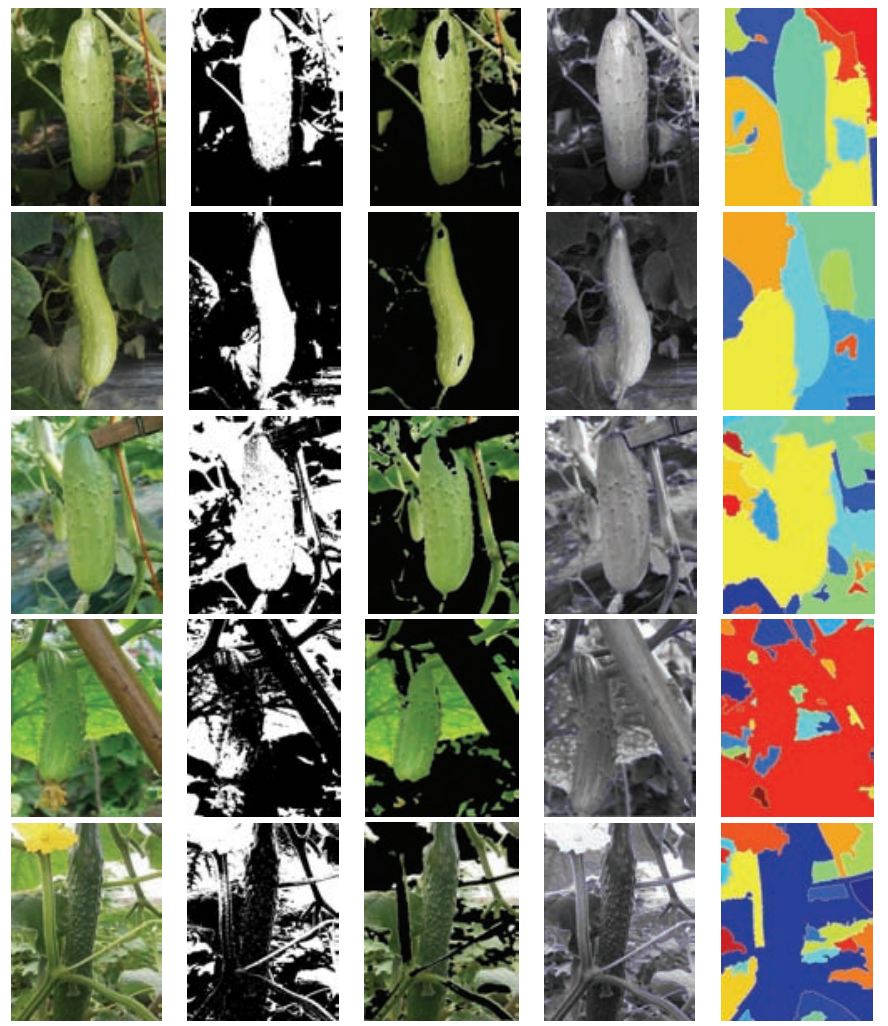

a
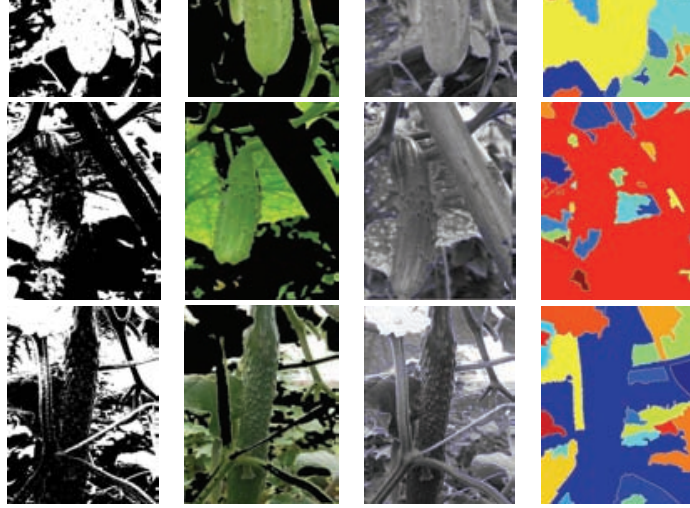

b

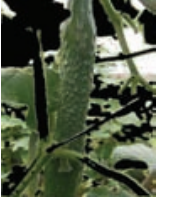

$\mathrm{c}$

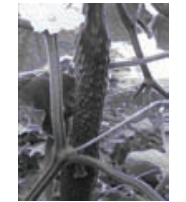

d

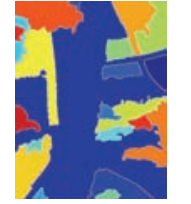

e
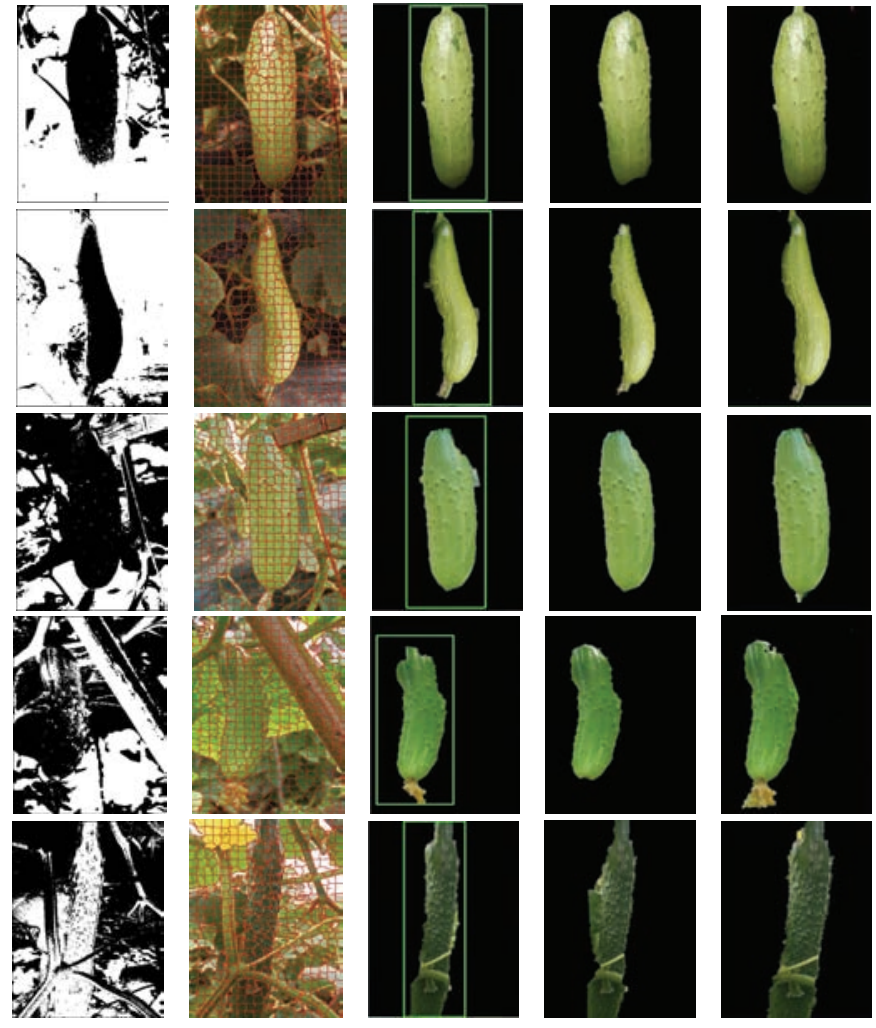

$\mathrm{h}$

i

j

Note: a. means original image; b. means OTSU method; c. means $K$-means method; d. means the Level Set method; e. means Watershed method; f. means Pulse Coupled Neural Network (PCNN); g. means Simple linear Iterative Clustering (SLIC) ${ }^{[30]}$; h. means traditional GrabCut algorithm; i. means improved GrabCut algorithm and j. means artificial segmentation samples.

Figure 6 Algorithms contrast

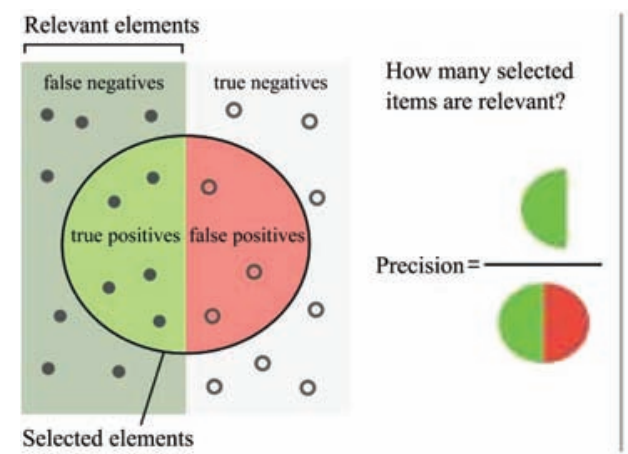

Figure 7 Evaluation indexes

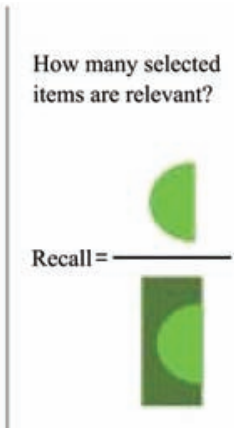

Table 1 Statistics of classification rate

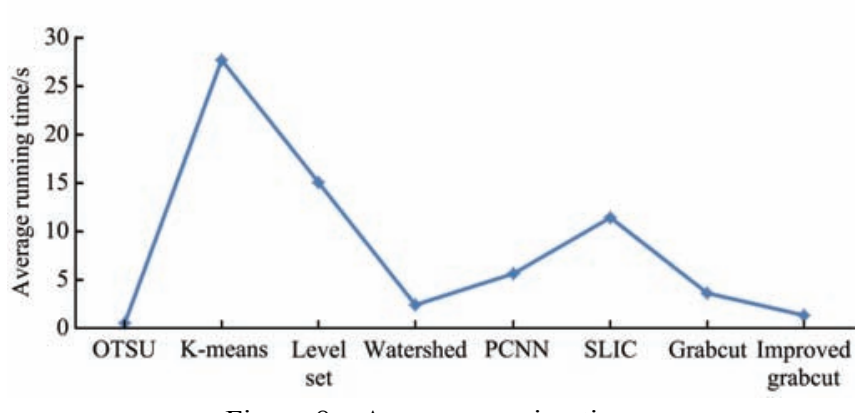

Figure 8 Average running time 
Table 2 Statistics of running time

Unit: s

\begin{tabular}{ccccccccc}
\hline Image & OTSU & $K$-means & Level Set & Watershed & PCNN & SLIC & Grabcut & Improved GrabCut \\
\hline 1 & 0.196 & 31.581 & 15.128 & 2.360 & 5.436 & 11.215 & 3.723 & 0.702 \\
2 & 0.323 & 29.928 & 16.727 & 2.235 & 5.489 & 11.232 & 3.606 & 1.257 \\
3 & 0.401 & 26.381 & 14.056 & 2.447 & 5.580 & 11.244 & 3.431 & 0.548 \\
4 & 0.480 & 27.721 & 12.505 & 2.694 & 5.944 & 11.260 & 3.626 & 1.693 \\
5 & 0.590 & 33.026 & 16.063 & 2.524 & 6.466 & 13.791 & 3.704 & 1.839 \\
6 & 0.697 & 29.345 & 19.088 & 2.881 & 5.742 & 11.373 & 3.355 & 1.398 \\
7 & 0.776 & 26.432 & 11.273 & 2.553 & 5.560 & 11.245 & 3.736 & 0.974 \\
8 & 0.849 & 21.928 & 14.424 & 2.222 & 5.642 & 11.233 & 3.571 & 2.05 \\
9 & 0.917 & 25.792 & 10.449 & 2.387 & 5.544 & 11.218 & 3.725 & 2.568 \\
10 & 0.991 & 25.005 & 20.681 & 2.495 & 5.619 & 11.187 & 4.014 & 0.994 \\
\hline
\end{tabular}

Note: iterative parameter $k=200$ in $K$-means method; iterative parameter $k=300$ in Level Set method; the optimal number is 500 and weight is 40 in SLIC method; and SLIC and GrabCut iteration number are both five times; all the operation time used above are the optimal segmentation time.

The improved GrabCut algorithm is optimal when comparing the image recognition effects of the eight methods, it is better than both the traditional GrabCut algorithm and the SLIC algorithm. Evaluation indexes in table 1 indicates that the improved GrabCut algorithm reaches up to $93.88 \%$ of Precision, $99.35 \%$ of the Recall rate, $96.53 \%$ of $F$-measure in all the contrast methods, and it can effectively control Error rate at 5.84\%. Table 2 shows that the improved GrabCut algorithm can shorten the average recognition time to $1.4023 \mathrm{~s}$. As for the stability of the statistics in tables, especially between the traditional GrabCut algorithm and improved GrabCut, the traditional GrabCut algorithm relied on the segmentation effect caused by human interaction heavily. Its stability was not good enough. For the split of the same image, the improved GrabCut realized the basic automatic segmentation, the data is within the scope allowed to float, which ensures the stability under control. Therefore, the improved GrabCut algorithm holds the perfect comprehensive segmentation effect, so that it can provide support for the next appearance quality detection.

\section{Cucumber appearance quality detection}

\subsection{Feature extraction}

After using the improved GrabCut algorithm to remove the complex background of cucumber image, the whole target color image is retained. The gray level co-occurrence matrix is used to extract the eight dimensional texture characteristic parameters (including energy, entropy, inertia moment, and correlation of the mean and standard deviation), and morphological operation is used to extract seven shape characteristic parameters as shown in Table 3. Then referring to the latest of the People's Republic of China agriculture industry standard NY/T 1587-2008 which gives three specifications of super fresh cucumber, primary, secondary and tertiary division mechanism has been set. In consideration of the parameters mean, maximum and minimum weight of the extracted shape and texture characteristics, rules for judging cucumber appearance quality are set.

\subsection{Quality detection and growth analysis}

Based on the above decision criteria for the quality, Figure 9 shows a designs window interface of cucumber appearance quality detection based on Matlab. It integrates the improved GrabCut image recognition algorithm, morphological processing for shape feature extraction, gray level co-occurrence matrix for texture feature extraction, and decision criteria for the quality detection and the growth analysis of short stick autumn cucumber.

\section{Table 3 Parameters for shape characteristics}

Unit: px

\begin{tabular}{cccccccc}
\hline Image & $\begin{array}{c}\text { Long } \\
\text { axis }\end{array}$ & $\begin{array}{c}\text { Short } \\
\text { axis }\end{array}$ & Area & Perimeter & $\begin{array}{c}\text { Narrow } \\
\text { extent }\end{array}$ & Circularity & Proportion \\
\hline 1 & 446.66 & 66.58 & 21170 & 996.24 & 0.851 & 0.268 & 0.141 \\
2 & 556.26 & 62.47 & 24888 & 1121.80 & 0.888 & 0.249 & 0.166 \\
3 & 491.51 & 94.24 & 32657 & 1012.32 & 0.808 & 0.400 & 0.218 \\
4 & 424.00 & 90.99 & 26623 & 970.04 & 0.785 & 0.356 & 0.177 \\
5 & 415.98 & 145.64 & 46619 & 1142.43 & 0.650 & 0.449 & 0.311 \\
6 & 490.46 & 43.38 & 16431 & 995.40 & 0.909 & 0.208 & 0.110 \\
7 & 278.30 & 27.28 & 5560 & 995.40 & 0.909 & 0.071 & 0.03 \\
8 & 391.32 & 43.06 & 12903 & 819.95 & 0.890 & 0.241 & 0.086 \\
9 & 381.47 & 79.26 & 18677 & 974.11 & 0.792 & 0.247 & 0.125 \\
10 & 385.96 & 97.78 & 28612 & 926.91 & 0.747 & 0.418 & 0.191 \\
11 & 481.38 & 48.32 & 16276 & 935.83 & 0.900 & 0.234 & 0.109 \\
12 & 476.49 & 143.67 & 53212 & 1122.48 & 0.698 & 0.531 & 0.355 \\
13 & 441.68 & 43.93 & 14374 & 886.68 & 0.901 & 0.230 & 0.096 \\
14 & 509.13 & 56.43 & 15302 & 1014.33 & 0.889 & 0.187 & 0.102 \\
15 & 346.46 & 28.41 & 6915 & 694.40 & 0.918 & 0.180 & 0.046 \\
16 & 350.10 & 77.41 & 16749 & 851.61 & 0.779 & 0.290 & 0.112 \\
17 & 493.49 & 67.80 & 18414 & 987.44 & 0.863 & 0.237 & 0.123 \\
18 & 334.70 & 81.96 & 20654 & 801.12 & 0.755 & 0.404 & 0.138 \\
19 & 467.24 & 41.74 & 15095 & 947.62 & 0.911 & 0.211 & 0.101 \\
20 & 504.37 & 126.32 & 45325 & 1104.27 & 0.750 & 0.467 & 0.302 \\
\hline & & & & & & & \\
\hline
\end{tabular}

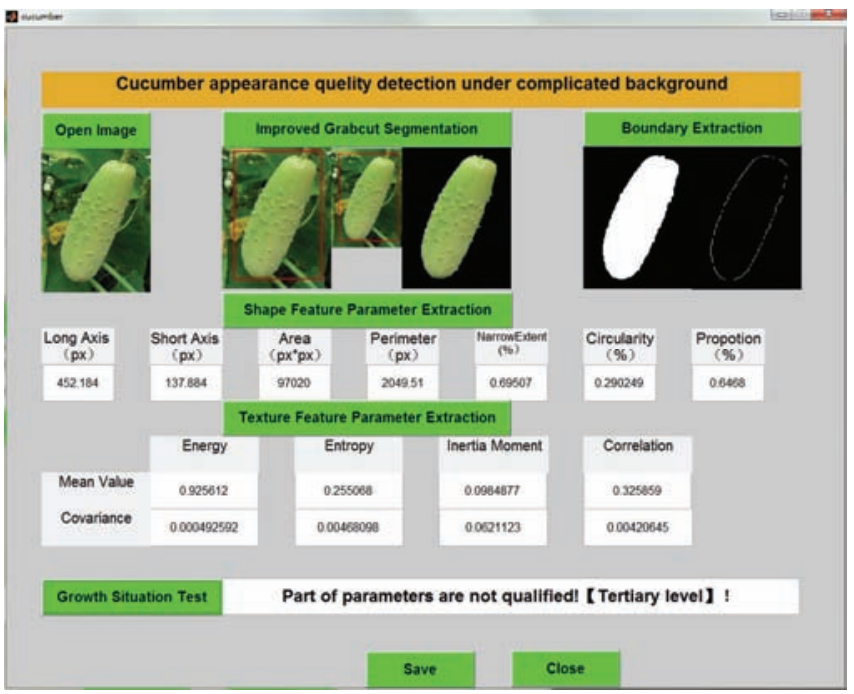

Figure 9 Cucumber appearance quality detection interface 
This paper chooses only 10 different types of cucumber image to make a verification about its function (as shown in Figure 10). Table 4 compares the test results of the interface with the actual artificial classification situation. The comparison analyzes that it can accurately distinguish autumn cucumber variety with white cucumber in image 1 and dense spines cucumber in image 6 . Cucumber in image 3 is precisely classified as a tertiary quality for

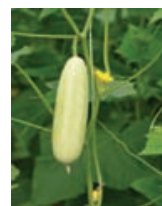

(1)

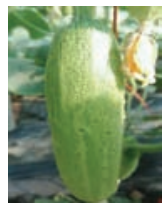

(2)

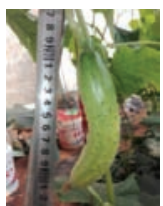

(3)

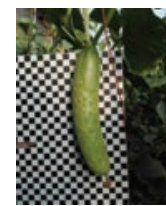

(4)

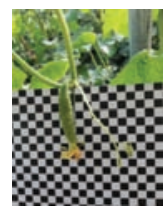

(5)

over-bending, and cucumber in image 5 is also divided into tertiary quality for immature fruit. Except image 2 which determines the misclassification for the geometric distortion, the other normal cucumber images are all divided into right level. The analysis shows that the quality criterion of the interface is effective and accurate.

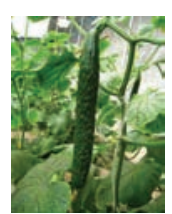

(6)

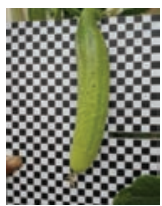

(7)

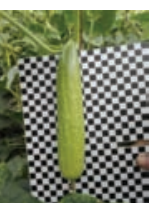

(8)

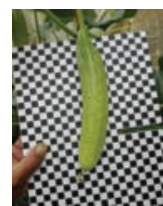

(9)

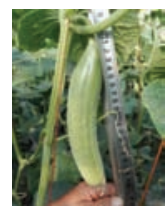

(10)

Figure 10 Cucumber appearance quality testing

Table 4 Comparison of quality detection

\begin{tabular}{|c|c|c|c|c|c|c|c|c|c|c|}
\hline Figure No. & $\begin{array}{l}\text { Testing } \\
\text { quality }\end{array}$ & $\begin{array}{l}\text { Actual } \\
\text { quality }\end{array}$ & $\begin{array}{c}\text { Testing } \\
\text { length/cm }\end{array}$ & $\begin{array}{c}\text { Actual } \\
\text { length/cm }\end{array}$ & $\begin{array}{l}\text { Error in } \\
\text { length/\% }\end{array}$ & $\begin{array}{c}\text { Testing } \\
\text { width/cm }\end{array}$ & $\begin{array}{c}\text { Actual } \\
\text { width/cm }\end{array}$ & $\begin{array}{c}\text { Error in } \\
\text { width } / \%\end{array}$ & $\begin{array}{c}\text { Bow } \\
\text { height } / \mathrm{cm}\end{array}$ & Details \\
\hline 1 & Tertiary & Tertiary & 15.3 & 15.9 & 4.4 & 4.8 & 5.1 & 5.9 & 0.03 & White cucumber \\
\hline 2 & Secondary & Primary & 15.8 & 22.4 & 29.5 & 6.8 & 4.8 & 41.7 & 0.05 & Image distortion \\
\hline 3 & Tertiary & Tertiary & 11.3 & 12.1 & 6.6 & 3.2 & 3.5 & 8.6 & 1.54 & Over bending \\
\hline 4 & Secondary & Secondary & 20.6 & 21.4 & 3.7 & 3.7 & 4.2 & 11.9 & 0.55 & Normal \\
\hline 6 & Tertiary & Tertiary & 23.4 & 25.2 & 7.1 & 3.1 & 3.3 & 6.1 & 0.06 & Dense spines cucumber \\
\hline 7 & Secondary & Secondary & 21.1 & 22.3 & 1.2 & 3.4 & 3.5 & 2.9 & 0.71 & Normal \\
\hline 8 & Primary & Primary & 22.6 & 24.4 & 7.4 & 4.2 & 4.4 & 4.5 & 0.02 & Normal \\
\hline 9 & Secondary & Secondary & 20.8 & 21.5 & 3.3 & 3.6 & 3.8 & 5.3 & 0.79 & Normal \\
\hline 10 & Secondary & Secondary & 19.1 & 20.3 & 6 & 3 & 3.3 & 9.1 & 0.93 & Normal \\
\hline
\end{tabular}

\section{Conclusions}

(1) The study improved GrabCut method which owned a strong applicability to realize the goal of cucumber nondestructive extraction under complex background. Its comprehensive evaluation index for quantitative analysis was superior to other methods, and it significantly improved recognition accuracy and efficiency of the algorithm.

(2) Under the complex background, image recognition effect depended on the complexity of the GrabCut algorithms and image size. Meanwhile, pre-segmentation was one of the effective methods to enhance image recognition effect.

(3) Quality detection results depended on the effect of image recognition and the distortion degree of image itself. Integrating the improved GrabCut algorithm, Morphological and grayscale symbiosis matrix, Cucumber appearance quality detection interface could solve the shape features and texture features extraction of autumn cucumber accurately.

(4) In the future, the study can provide technical support for subsequent cucumber quality accurate classification, picking robot and fruit and vegetable identification on mobile terminal.

\section{Acknowledgments}

This study was financially supported by the National Science and Technology Support Project (2014BAD04B05), Transformation and popularization project of agricultural scientific and technological achievements in Tianjin - "Integrated application of core information technology for early warning, diagnosis and prevention of greenhouse vegetable diseases" (201704070).

\section{[References]}

[1] Li Z G, Thomas C. Quantitative evaluation of mechanical damage to fresh fruits. Trends in Food Science \& Technology, 2014; 35(2): 138-150.

[2] Dafni A, Moses Y, Avidan S, Dekel T. Detecting moving regions in CrowdCam images. Computer Vision and Image Understanding, 2017; 160: 36-44.

[3] Loutfi A, Coradeschi S, Mani G K, Shankar P, Rayappan J B B. Electronic noses for food quality: A review. Journal of Food Engineering, 2015; 144: 103-111.

[4] Boykov Y Y, Jolly M P. Interaction graph cuts for optimal boundary \& region segmentation of objects in ND images. Computer Vision, IEEE, 2001.

[5] Rother C, Kolmogorov V, Blake A. "GrabCut" - interative foreground extraction using iterated graph cuts. ACM Trans on Graphics, 2004; 23(3): 307-312.

[6] Jahangiri M, Heesch D. Modified GrabCut for unsupervised object segmentation. $16^{\text {th }}$ International Conference on Information Processing (ICIP), 2009; pp.2389-2392. DOI: 10.1109/ICIP.2009.5414500

[7] Kim G, Sim J Y. Depth guided selection of adaptive region of interest for GrabCut-based image segmentation. Asia-Pacific Signal and Information Processing Association Annual Summit and Conference (APSIPA), 2016.

[8] Kang S. Object Segmentation using mean-shift with grid-mask for grab cut algorithm. International Journal of software Engineering and its Applications, 2014; pp.409-416.

[9] Poullot S, Satoh S. VabCut: A video extension of GrabCut for unsupervised video foreground object segmentation. International Conference on Computer Vision Theory and Applications, IEEE, 2014; pp.362-371.

[10] Xu S X, Zhu Q Y. Seabird image identification in natural scenes using Grabcut and combined features. Ecological Informatics, 2016; 33: 24-31.

[11] Lee G S, Lee S H, Kim G O, Park J H, Park Y S. A modified Grabcut using a clustering technique to reduce image noise. Symmetry, 2016; 8(7): 64. https://doi.org/10.3390/sym8070064 
[12] Huang L L. Study on grab cut and its improved algorithm. Software Guide, 2015; 5: 65-67. (in Chinese)

[13] Yang S B, Li L M, Huang Y Q. Adaptive background image segmentation algorithm based on GrabCut. Computer System Application, 2017; 26(2): 174-178. (in Chinese)

[14] Hu Z L, Guo M. Fast segmentation in color image based on SLIC and GrabCut. Computer Engineering and Applications, 2016; 52(2): 186-190. (in Chinese)

[15] Cao Y, Jiang Y, Gao H, Chen H, Fang X, Mu H, Tao F. Development of a model for quality evaluation of litchi fruit. Computers and Electronics in Agriculture, 2014; 106: 49-55.

[16] Elortondo F J P, Ojeda M, Albisu M, Salmerón J, Etayo I, Molina M. Food quality certification: An approach for the development of accredited sensory evaluation methods. Food Quality and Preference, 2007; 18(2): 425-439.

[17] Zhuo C, Chen D. Evaluation factors of Kumquat quality determined by the principal component analysis. Subtropical Agriculture Research, 2011; 7(2): 132-135.

[18] Zhang B, Huang W, Li J, Zhao C, Fan S, Wu J, Liu C. Principles, developments and applications of computer vision for external quality inspection of fruits and vegetables: A review. Food Research International, 2014; 62: 326-343.

[19] Zhang H, Yang S, Joyce D C, Jiang Y, Qu H, Duan X. Physiology and quality response of harvested banana fruit to cold shock. Postharvest Biology and Technology, 2010; 55(3): 154-159.

[20] Zhuo C, Chen D. Evaluation factors of Kumquat quality determined by the principal component analysis. Subtropical Agriculture Research, 2011; 7(2): 132-135

[21] Zhang H, Han T, Wang Y, Li L. Selection of factors for evaluating peach
(Prunus persica) fruit quality. Transactions of the CSAE, 2006; 22(8): 235-239.

[22] Chen Q, Zhang C, Zhao J, Ouyang Q. Recent advances in emerging imaging techniques for non-destructive detection of food quality and safety. TrAC Trends in Analytical Chemistry, 2013; 52: 261-274.

[23] Abbaszadeh R, Rajabipour A, Ahmadi H, Mahjoob M J, Delshad M. Prediction of watermelon quality based on vibration spectrum. Postharvest Biology and Technology, 2013; 86: 291-293.

[24] Cristina M, Laura F, Riccardo G, Ernestina C, Riccardo R. GLCM, an image analysis technique for early detection of biofilm. Journal of Food Engineering, 2016; 185: 48-55.

[25] Hua S Y, Shi P. GrabCut color image segmentation based on region of interest. International Congress on Image and Signal Processing, IEEE, 2014.

[26] Rother C, Kolmogorov V. An experimental comparison of min-cut/max-flow algorithms for energy minimization in vision. Tissue Engineering, 2005; 11(12): 1631-1639.

[27] Kim K S, Yoon Y J, Kang M C, Sun J Y, Ko S J. An improved GrabCut using a saliency map. IEEE 3rd Global Conference on Consumer Electronics (GCCE). 2014.

[28] Guo C X, Li Z B, Qiao X, Li C, Yue J. Image segmentation of underwater sea cucumber using GrabCut with saliency map. Transactions of the CSAM, 2015; 46(S1): 147-152. (in Chinese)

[29] Taichi I, Shunji K. Evaluation of bending behavior of flexible pipe using digital image processing. Procedia Engineering, 2017; 171: 1272-1278.

[30] Achanta R, Shaji A, Smith K, Lucchi A, Fua P, Süsstrunk S. SLIC superpixels compared to state-of-the-art superpixel methods. IEEE Transactions on Pattern Analysis and Machine Intelligence, 2012; 34(11): 2274-2282. DOI: 10.1109/TPAMI.2012.120 